\title{
ТРЕБУЕМОЕ И ДОБРОВОЛЬНОЕ РАСКРЫТИЕ ИНФОРМАЦИИ ПО РИСКАМ В ФИНАНСОВОЙ ОТЧЕТНОСТИ: СТИМУЛЫ И ПРЕПЯТСТВИЯ *
}

\author{
(c) 2019 Листопад Екатерина Евгеньевна \\ кандидат экономических наук, доцент Департамента учета, анализа и аудита \\ Финансовый университет при Правительстве Российской Федерации, Россия, Москва \\ E-mail: eelistopad@mail.ru
}

В предлагаемой статье на практических примерах предложена и раскрыта возможность отражения в финансовой отчетности хозяйственных рисков, выделены стимулы и препятствия отражения информации о рисках, приведены преимущества добровольного раскрытия информации.

Ключевые слова: отчет о рисках, экономические выгоды, риски утраты и случайной гибели, право собственности, рыночный риск, кредитный риск, риск ликвидности, оценка риска.

Рискология является областью, которая вызвала большой научный интерес в последние годы. Основными направлениями исследований, которые используются в многочисленных публикациях, являются оценка финансового риска хозяйствующего субъекта, налоговые риски и аудиторские риски. Многими авторами в настоящее время разработаны различные методики оценки рисков, как правило, инвестиционных и финансовых, а также связанных со спецификой предпринимательской деятельности в различных сферах. Многие исследования посвящены разработке механизмов управления этими рисками, однако, мало внимания уделено разработкам учетно-аналитическим механизмам хозяйственных рисков, не исследованы вопросы универсализации системы учета и анализа совокупности предпринимательских рисков для целей их оптимизации по количеству, качеству и затратам времени на процедуры оценки и принятия управленческих решений.

Рыночная экономика требует раскрытия информации и о хозяйственных рисках в отчетах компаний. Это призвано показать качественный уровень раскрытия информации в общем объеме отчетов компании. Оно может использоваться для демонстрации соблюдения нормативных положений, если элементы риска выбраны таким образом, или, наоборот, оно может использоваться для демонстрации уровня добровольного раскрытия информации $[2,3,5,6]$. Однако, данной проработки настоящей проблемы недостаточно в связи с постоянным реформированием бухгалтерского учета и отчетности. Элементы, выбранные для отражения хозяй- ственного риска в отчетности, вероятно, будут довольно небольшим поднабором совокупности всех элементов рисков, которые могут быть раскрыты компанией. Количество рисков, которые могут быть раскрыты компанией, очень велико, если не бесконечно:

- налоговые риски;

- риски неоплаты по договорам;

- валютные риски;

- риски чрезвычайных ситуаций;

- политические;

- странновые;

- финансовые риски и др.

Наиболее распространенные классификации в экономической литературе, предусматривают деление рисков по группам, представленным на рисунке 1 . Поэтому полезность раскрытия информации о рисках в решающей степени зависит от выбора элементов, которые будут включены в отчетность по рискам.

Считаем, что основной задачей в настоящее время является исследование достоверности полученной информации о рисках. Информация о хозяйственных рисках может раскрываться компаниями несколькими способами. Основным средством раскрытия информации является годовая отчетность. Кроме того, могут быть промежуточные и квартальные отчеты, а также проспекты, отчеты сотрудников, объявления на бирже и другие печатные материалы.

Информация также может раскрываться более неофициально, на встречах с аналитиками или в телефонных разговорах. Однако, в настоящем исследовании остановимся только на информации о рисках, раскрываемой в годовой

BAK 08.00.05, 08.00.14 

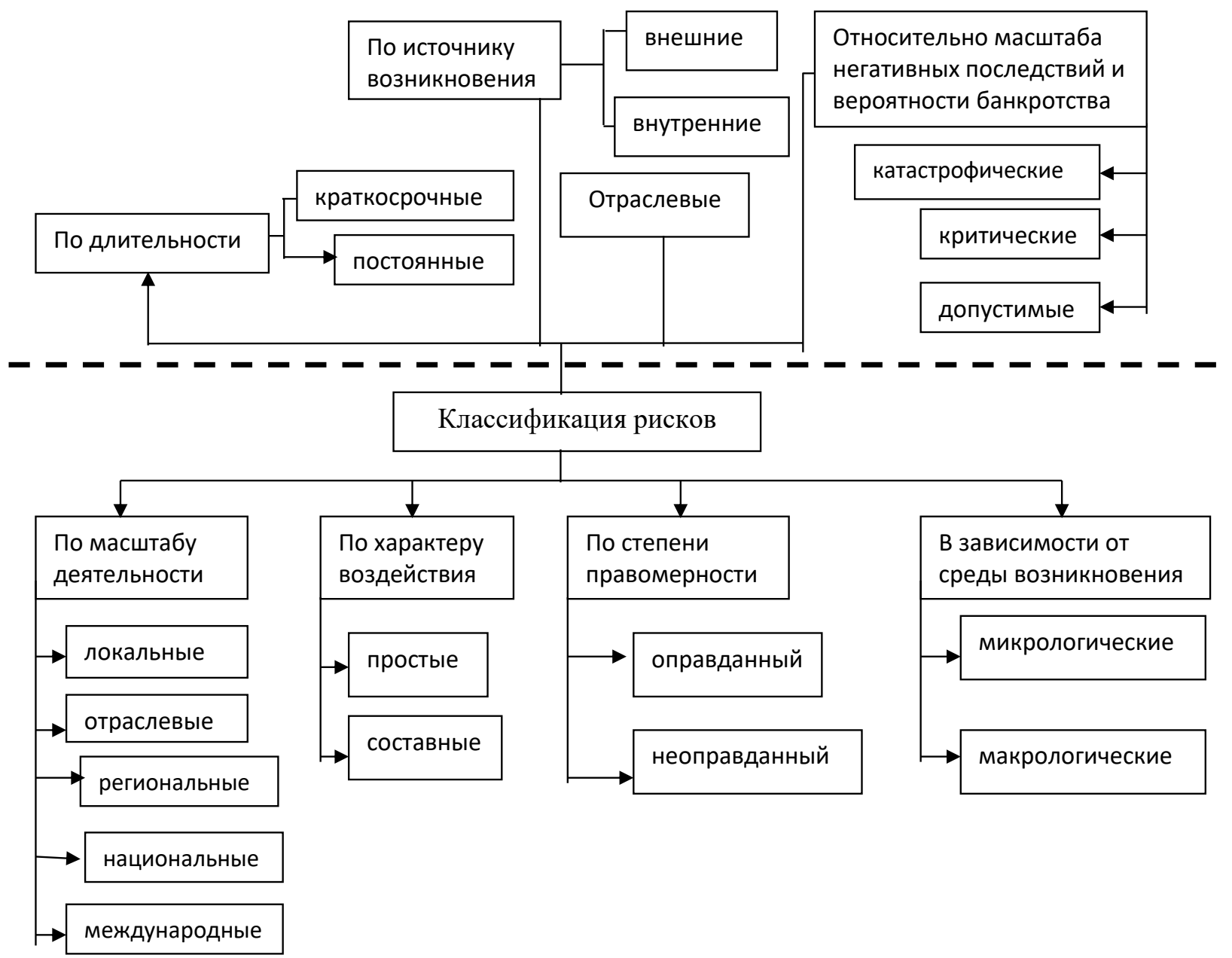

Рис. 1. Классификация рисков

финансовой отчетности.

Эту информацию предлагаем разделить на две широкие категории:

1. требуемое раскрытие;

2. добровольное раскрытие.

Требуемое раскрытие информации устанавливается законом, профессиональными правилами и листинговыми требованиями фондовых бирж. Степень соблюдения компаниями законодательных и нормативных требований зависит от строгости или слабости государственных, профессиональных и других регулирующих органов.

Например, в информации Министерства финансов Российской Федерации N П3-9/2012 «О раскрытии информации о рисках хозяйственной деятельности организации в годовой бухгалтерской отчетности» предложены рекомендации Минфина о раскрытии в годовой бухгалтерской отчетности организации показателей и пояснений о потенциально существенных рисках хозяйственной деятельности, которым подвержена организация; информации с целью формирования полного представления о финансовом положении организации, финансовых результатах ее деятельности и изменениях в ее финансовом положении. Раскрытие указанной информации является одной из составляющих системы внутреннего контроля совершаемых фактов хозяйственной жизни организации [1].

Так, в данном документе Минфин предлагает раскрывать информацию следующих группах рисков:

- рыночные риски (табл. 1);

- кредитные риски (табл. 2);

- риски ликвидности (табл. 3).

Если у организации имеется солидарная ответственность, например, по сделкам РЕПО, по опционам, по переданным векселям, то она должна обособленно проанализировать риски, которые связаны с передаваемыми активами. При этом организация должна обеспечить соот- 
Таблица 1. Объем раскрываемой информации в отчетности по группе рыночных рисков [1]

\begin{tabular}{|c|c|c|c|}
\hline Группа риска & Причина риска & Примеры риска & $\begin{array}{c}\text { Действия субъекта/объем } \\
\text { раскрываемой информации } \\
\text { в отчетности }\end{array}$ \\
\hline Рыночные риски & $\begin{array}{l}\text { К причинам возникновения } \\
\text { риска можно отнести воз- } \\
\text { можные неблагоприятные } \\
\text { для организации послед- } \\
\text { ствия в случае изменения } \\
\text { рыночных параметров }\end{array}$ & $\begin{array}{l}\text { Пример рыночных рисков: } \\
\text { • цены на товары, гото- } \\
\text { вую продукцию, работы, } \\
\text { услуги; } \\
\text { • цены на ценные бумаги, } \\
\text { драгоценные металлы и } \\
\text { др.; } \\
\text { • процентные ставки; } \\
\text { • курсы иностранных валют }\end{array}$ & $\begin{array}{l}\text { По состоянию на отчет- } \\
\text { ную дату хозяйствующий } \\
\text { субъект должен проанали- } \\
\text { зировать и определить свою } \\
\text { чувствительность к каждому } \\
\text { подвиду рыночных рисков, } \\
\text { которым он может быть } \\
\text { подвержен }\end{array}$ \\
\hline
\end{tabular}

ветствующий расчет, подтверждающий достоверность оценки данной группы рисков. Далее исследователи должны отразить финансовый эффект, который потенциально могут оказать рыночные риски на финансовое положение, на финансовые результаты, на движение денежных потоков организации, т.е. на изменение соответствующей переменной, от которой зависит уровень риска.

Обособленно раскрывается также информация о хеджировании указанных рисков.

В табл. 2 представлен объем раскрываемой обязательной информации по группе кредитных рисков.

При этом максимальный размер потенциального кредитного риска, а также методика его определения должны раскрываться обособленно.

Далее предлагаем рассмотреть добровольное раскрытие информации сверх минимума, предложенного Минфином, которое может возникать в тех случаях, когда корпоративное восприятие связанных с этим выгод перевешивает издержки.
Информация о рисках в годовом отчете состоит из качественных и количественных данных. Количественные данные являются как финансовыми, так и нефинансовыми. Для формирования количественных финансовых данных исследователи разработали методы оценки риска, основанные на их субъективных взглядах, и, как правило, данные методики основываются на потребности «образованного инвестора».

Считаем, что цифры нельзя рассматривать изолированно, как имеющие какое-либо информационное наполнение, они должны сопровождаться пояснительными словами. Компании отличаются сложностью своей деятельности, структурой и иерархией, а также документооборотом. Поэтому многонациональные и многопрофильные компании должны раскрывать больше информации, чем более простые организации.

Вместе с тем необходимо четко признавать проблему, связанную с оценкой достоверности раскрытия финансовой информации о рисках, потому что такое раскрытие финансовой инфор-

Таблица 3. Объем раскрываемой информации в отчетности по группе рисков ликвидности [1]

\begin{tabular}{|c|c|c|c|}
\hline Риск ликвидности & $\begin{array}{l}\text { К причинам возникнове- } \\
\text { ния риска можно отнести } \\
\text { отсутствие возможности } \\
\text { организации своевре- } \\
\text { менно и в полном объеме } \\
\text { погасить имеющиеся на } \\
\text { отчетную дату финансо- } \\
\text { вые обязательства }\end{array}$ & $\begin{array}{l}\text { Финансовые обязатель- } \\
\text { ства анализируются по } \\
\text { срокам погашения отно- } \\
\text { сительно отчетной даты в } \\
\text { соответствии с условиями } \\
\text { получения (заключенными } \\
\text { договорами, условиями } \\
\text { размещения облигаций, } \\
\text { условиями выпуска вексе- } \\
\text { лей). }\end{array}$ & $\begin{array}{l}\text { В отчетности как минимум } \\
\text { должна быть раскрыта инфор- } \\
\text { мация: } \\
\text { • о приведенной стоимости } \\
\text { финансовых обязательств; } \\
\text { - о справедливой стоимости } \\
\text { финансовых обязательств, } \\
\text { если она отличается от } \\
\text { приведенной стоимости и } \\
\text { практически определима на } \\
\text { отчетную дату; } \\
\text { - о выданных обеспечениях; } \\
\text { - об имеющихся ограничениях } \\
\text { по использованию в заплани- } \\
\text { рованных целях неоплачен- } \\
\text { ных активов; } \\
\text { - риск начала процедуры } \\
\text { банкротства или ликвидации } \\
\text { организации. }\end{array}$ \\
\hline
\end{tabular}


Таблиц̧а 2. Объем раскрываемой информации в отчетности по группе кредитных рисков [1]

\begin{tabular}{|c|c|c|c|}
\hline Кредитные риски & $\begin{array}{l}\text { К причинам возникнове- } \\
\text { ния риска можно отнести } \\
\text { возможные неблагопри- } \\
\text { ятные для организации } \\
\text { последствия при неиспол- } \\
\text { нении или ненадлежащем } \\
\text { исполнении обязательств } \\
\text { другими лицами }\end{array}$ & $\begin{array}{l}\text { Примеры кредитных рисков: } \\
\text { • последствия при ненадле- } \\
\text { жащем исполнении другими } \\
\text { лицами обязательств по } \\
\text { предоставленным заемным } \\
\text { средствам; } \\
\text { • последствия при ненадле- } \\
\text { жащем исполнении други- } \\
\text { ми лицами обязательств в } \\
\text { форме покупки векселей и } \\
\text { облигаций; } \\
\text { последствия при ненадле- } \\
\text { жащем исполнении другими } \\
\text { лицами обязательств при } \\
\text { предоставлении отсроч- } \\
\text { ки и рассрочки оплаты за } \\
\text { проданные товары, работы } \\
\text { или услуги }\end{array}$ & $\begin{array}{l}\text { В частности, раскрывается } \\
\text { информация: } \\
\text { • о сомнительной (в том } \\
\text { числе просроченной) } \\
\text { дебиторской задолжен- } \\
\text { ности; } \\
\text { - об обесценившихся пре- } \\
\text { доставленных займах; } \\
\text { - о приведенной и справед- } \\
\text { ливой стоимости долго- } \\
\text { вых финансовых вложе- } \\
\text { ний на отчетную дату. } \\
\text { Раскрывается также инфор- } \\
\text { мация о положении деби- } \\
\text { тора }\end{array}$ \\
\hline
\end{tabular}

мации является субъективным понятием, которое не может быть измерено непосредственно, т.к. не обладает присущими характеристиками, по которым можно определить качество.

С учетом характера риска, при раскрытии информации о рисках, необходимо учитывать два критерия, которые обычно используются в социальных науках при оценке показателей. Этими критериями являются:

1) критерий надежности;

2) критерий валидности.

Надежность показателей риска. Оценка риска, присвоенная компанией, может считаться надежной, если результаты могут быть воспроизведены другим исследователями (пользователями). Поскольку оценка рисков извлекается из печатных годовых отчетов, которые остаются неизменными с течением времени, нет никаких препятствий для их повторения и проверки. Однако, можно столкнуться с рядом практических проблем при оценке рисков. В частности, существуют проблемы частичных оценок и решений относительно того, действительно ли нераскрытая информация о рисках применима к конкретной компании. Для того, чтобы последующий исследователь мог повторить «рисковый» скоринг (анкетирование для оценки рисков), необходимы четкие инструкции по проблемным областям. Поэтому для оценки надежности информации о рисках считаем необходимым включать инструкцию наряду с перечнем информации о рисках и их соответствующих элементах. Аналогичным образом, необходимо предоставить доказательную базу, которая применялась при принятии решения о том, имеет ли данный вид риска отношение к конкретной компании.
Помимо этого в отчетности по рискам необходимо по нашему мнению предоставлять доказательства того, что меры его раскрытия являются действительными и надежными. Для этого считаем необходимым конкретизировать оперативные процедуры, в соответствии с которыми были получены эмпирические данные о рисках.

Оценка риска может считаться действительной, если она означает то, что предполагали исследователи. Иными словами, имеют ли показатели риска какое-либо значение в качестве меры раскрытия информации? Этот вопрос не рассматривался большинством цитируемых авторов. Поэтому, считаем, что исследователи должны представить доказательства того, что оценка риска является действительной и надежной.

Тот факт, что ни одна конкретная методика оценки риска не пользуется популярностью у исследователей, иллюстрирует еще один аспект, а именно: проблемы обоснованности. Большинство исследователей адаптируют существующие методы оценки для удовлетворения своих собственных потребностей. Это попытка создать «свою» информацию о рисках, которая действительна в конкретной исследовательской среде. Желательность разработки стандартного многоцелевого подхода к оценке риска прямо не рассматривалась. Однако, на международном уровне рассматривают проблемы разработки единой методики оценки риска, подходящего для межнациональных компаний. Любая попытка создать универсальную методику оценки риска для раскрытия информации в отчетности вряд ли будет иметь смысл, если международное 
сообщество не достигнет такого соглашения. Нет «международного оператора рынка капитала», поэтому получить согласованную на международном уровне модель восприятия информации невозможно, и трудно оценить актуальность для пользователей информационных элементов раскрытия информации. Если никто не знает о потребностях такого пользователя, было бы трудно измерить качество информации, содержащейся в годовом отчете компании.

Различные группы пользователей могут иметь тенденцию рассматривать различные элементы отчетности как важные: очевидно, что один класс пользователей будет придавать предмету разные веса, чем другой класс пользователей. Поэтому при составлении отчетности по рискам необходимо описать информационные потребности основных групп участников таких, как финансовые аналитики и сотрудники. Информационные потребности различных групп могут пересекаться, хотя основной акцент может отличаться. Например, финансовые аналитики будут более заинтересованы в раскрытии информации о финансовых результатах и потенциальной прибыли, тогда как группы сотрудников будут более заинтересованы в раскрытии информации, касающиеся риска изменений условий занятости, риска снижения оплаты труда и риска сокращения штата. Таким образом, выбор элементов авторами часто зависит от ориентации риска на группы пользователей: поскольку мера степени раскрытия связана как с характером пользователя, так и с его целью, необходимо выбрать группу пользователей и цель.

Предположение ориентации группы пользователей на определенную методику оценки риска или выбор его элементов может иметь интуитивную направленность. По сути, аналитики пытаются выбрать те элементы или ту методику, которые группа пользователей считает важными. Тогда возникает проблема формирования консенсуса группы. С одной стороны, это правильный путь. Однако, если рассматривать проблему консенсуса в группах пользователей, то можно поставить под сомнение предположение о том, что восприятие пользователей может быть вызвано исследованием, т.е. научным подходом. По нашему мнению, консенсус может касаться трех взаимосвязанных вопросов, причем только один из них может быть изучен одновременно:

- стабильности восприятия;

• однородности групп пользователей;
- однородности элементов раскрытия информации.

Восприятие является стабильным, если все пользователи придерживаются одинаковых взглядов на уровень важности элемента, независимо от их различных потребностей в элементе. Группа пользователей однородна, если в ней нет существенных различий по степени важности элемента. Элемент однороден, если он означает одно для каждого члена группы пользователей и другое для каждого члена другой группы пользователей. Но информационные потребности пользователей различаются (и должны различаться) внутри фирмы и между фирмами, внутри регионов и между регионами, внутри отрасли и между отраслями, внутри страны и между странами, поскольку элементы информации способны различать пользователей, а представления пользователей об этих элементах должны быть стабильными.

В годовых отчетах необходимо подчеркнуть потребности группы пользователей при выборе элементов риска финансовым аналитиком. Это согласуется с большим количеством бухгалтерских отчетов, которые концентрируются на самой сложной информации для определенных продвинутых пользователей, хотя отчетность может представлять интерес для общества в целом, если использует другой, более широкий подход, который не будет направлен на интересы каких-либо конкретных групп пользователей.

Цель, которая формулируется при разработке системы учета рисков в организации, также имеет некоторое отношение к выбору элементов риска и методам его оценки.

Для разработки элементов риска используется обзор соответствующей литературы, а для выбора наиболее важных - профессиональное суждение.

Еще одна проблема, которая возникает, заключается в том, что некоторые элементы раскрытия информации могут быть неприменимы к конкретной компании. Очевидно, что компании не должны наказываться за неразглашение информации в этом случае. В некоторых случаях эта проблема может быть устранена путем обеспечения того, чтобы все элементы раскрытия были актуальны для всех компаний в выборке. Рекомендуем в отчетности по рискам формировать информацию, даже если данный вид риска или данная методика его оценки не использовалась, т.к. в случаях неразглашения не всегда ясно, 
является ли данный пункт актуальным или нет. Например, если условные обязательства не раскрываются, означает ли это, что они отсутствуют или что компания отказывается раскрывать их? В этом случае необходимо ознакомиться со всем годовым отчетом, а после чего принять соответствующее решение о том, не был ли тот или иной риск не раскрыт или он не имеет отношения к компании.

При проведении исследования стало очевидным, что связь между уровнем раскрытия информации о рисках и отдельными характеристиками компаний часто взаимосвязана.

Вполне естественно предположить, что крупные компании будут раскрывать больше информации, чем малые компании. Существует ряд причин, по которым можно ожидать положительной взаимосвязи между размером активов корпорации и качеством раскрытия информации. Затраты на накопление подробной информации относительно выше для небольших компаний, в то время как в более крупных компаниях эта информация уже накапливается для целей внутренней отчетности. Во-вторых, руководство более крупной корпорации с большей вероятностью осознает возможные преимущества более эффективного раскрытия информации, такие, как облегчение сбыта ценных бумаг и облегчение финансирования. Небольшие корпорации обычно не собирают деньги на рынке ценных бумаг и, следовательно, не могут реализовать возможные преимущества лучшего раскрытия информации. Наконец, более мелкие корпорации с большей вероятностью считают, что полное раскрытие информации может поставить под угрозу их конкурентное положение.

Существует несколько причин для того, чтобы ожидать положительной связи между размером фирмы и степенью раскрытия информации, в том числе информации о рисках. Крупные предприятия несут политические риски. Поэтому, для их нивелирования они используют такие механизмы, как составление социальной отчетности, для предотвращения дальнейшего регулирования или национализации. Еще одной причиной более широкого раскрытия информации крупными фирмами является то, что такие предприятия, вероятно, будут более сложными с точки зрения товарных зон и географического положения. Последующее представление сложной управленческой информации облегчает подготовку более сложной внешней отчетности.
Таким образом, размер корпорации не приводит к различиям в уровнях раскрытия информации. Скорее всего, у крупной компании больше шансов иметь первопричины для более широкого раскрытия информации, чем у небольшой компании.

Размер компании не является единственной характеристикой, связанной с раскрытием информации. Необходимо отметить, что на качество раскрытия информации во всех странах мира в значительной степени влияют листинговые требования различных фондовых бирж и правила отчетности комиссии по ценным бумагам и биржам. Таким образом, котирующиеся на бирже компании, скорее всего, раскроют больше, чем незарегистрированные компании. Хотя, например, Стокгольмская Фондовая биржа не требует дополнительного раскрытия информации, все разумно крупные предприятия в Швеции подпадают под одинаковые требования к раскрытию информации. Однако шведские компании получают листинги на зарубежных фондовых биржах и подпадают под действие более чем одного набора требований о раскрытии информации. Это может привести к тому, что они будут раскрывать большее число статей в своих корпоративных отчетах. Множественный листинг может указывать на большую потребность во внешнем капитале, и эта потребность является возможным объяснением увеличения раскрытия информации.

Необходимость привлечения нового капитала может быть объяснительным фактором, ведущим к увеличению раскрытия информации с течением времени. Малые фирмы увеличивают раскрытие информации при привлечении новых финансовых средств на фондовом рынке, в то время как для более крупных фирм таких отношений, как представляется, не существует.

В частности, можно предположить, что финансовый рычаг может влиять на добровольное раскрытие финансовой информации, влияя на величину агентских расходов и/или расходов на заключение контрактов между управляющим и внешним собственником.

Изучение взаимосвязи между внутренними характеристиками конкретной фирмы и степенью раскрытия информации может быть расширено, с тем, чтобы охватить внешнюю характеристику конкретной фирмы, рыночные показатели котируемых акций фирмы. Более широкое раскрытие информации должно 
уменьшить неопределенность инвесторов и, следовательно, может способствовать оценке рынком риска, связанного с конкретной акцией или компанией.

Еще одним из аспектов добровольного раскрытия информации является сфера социальной и экологической отчетности. Можно ожидать, что компании, широко раскрывающие добровольную информацию в этих областях, будут также проводить соответствующую социальную и экологическую политику. Однако, фирмы, раскрывающие информацию по рискам, будут проводить политику по их снижению. При этом существуют исследования, посвященные взаимосвязи между социальной эффективностью и социальной открытостью, которые дали неоднозначные результаты: изучив конкретное содержание корпоративной экологической информации, используя процедуру индексации и сравнивая это с фактическими экологическими показателями, не было обнаружено никакой связи между разглашением и исполнением. В результате можно сделать предварительный вывод, что формирование отчетности по рискам, при условии надежности и достоверности её информация, не дает прямой связи с принятием управленческих решений по минимизации рисков, и усилению внутреннего контроля с целью их ликвидации.

Использование информации о рисках в качестве повышения достоверности отчетности в целом, в настоящее время игнорируется.
В заключение настоящего исследования можно сказать, что аналитики могут использовать раскрытие информации о рисках в качестве средства измерения достоверности и качества корпоративной отчетности. С одной стороны, получение обоснованных результатов является необходимым, но недостаточным условием признания методики полезной в эмпирических исследованиях.

Таким образом, из обзора литературы и практики становится очевидно, что формирование отчетности по рискам является сложным вопросом, который, как правило, предполагает субъективное суждение со стороны исследователей. Кроме того, во многих случаях компаниям, как представляется, также предполагается субъективное суждение. В социальных науках многие общие исследовательские инструменты вызывают споры и разногласия. Измерение раскрытия информации в компании не может осуществляться точным научным способом. Субъективность исследователя не может быть полностью удалена, и не разумно ожидать, что этого можно избежать. Таким образом, значение полученных результатов раскрытия информации и их последующее использование при проверке гипотез нельзя рассматривать некритично. Здесь актуальны усилия исследователя по минимизации субъектности и разработке более объективной методики оценки и раскрытия информации о рисках в отчетности.

\section{Библиографический список}

1. Информации Министерства финансов Российской Федерации N П3-9/2012 «О раскрытии информации о рисках хозяйственной деятельности организации в годовой бухгалтерской отчетности».

2. Толстова А.С. Бухгалтерские риски и их влияние на достоверность бухгалтерской отчетности: диссертация кандидата экономических наук: 08.00.12 / Толстова А. С.; [Место защиты: Марийс. гос. техн. ун-т]. Нижний Новгород. 2009. 201 с.

3. Ахметова А. Ж. Финансовая неопределенность и риск в финансовой системе//Евразийский национальный университет им. Л.Н. Гумилева. Международный экономический форум. Казахстан. URL: http://www. be5. biz/ekonomika1/r2010/01504. Htm.

4. Кабанова Е.А., Трегуб И.В. Создание оптимального инвестиционного портфеля// Международный студенческий научный вестник. 2015. № 1 .

5. Фёдорова Л.И., Васильева Н.В. Особенности бухгалтерских рисков и способы их предотвращения // Образование и наука в современных условиях. Издательство: ООО «Центр научного сотрудничества «Интерактив плюс» (Чебоксары), № 1, 2015 г.с. 279-283

6. Шевелев А.Е. Риски в бухгалтерском учете на современном предприятии // Вестник Южно-Уральского государственного университета. Серия: Экономика и менеджмент. 